Check for updates

Cite this: RSC Adv., 2019, 9, 27574

\title{
Improved lithium ion dynamics in crosslinked PMMA gel polymer electrolyte $\uparrow$
}

\author{
Ava Hosseinioun, ${ }^{a}$ Pinchas Nürnberg, ${ }^{\mathrm{b}}$ Monika Schönhoff, (D) *b Diddo Diddens ${ }^{* a}$ \\ and Elie Paillard (D) *a
}

Since PMMA-based gel polymer electrolytes could substitute PVDF-HFP based gels currently used in Li-ion batteries at lower financial and environmental costs, we investigate here the solvation and transport properties of the lithium ions in a crosslinked PMMA-based gel polymer electrolyte by a combination of thermal and electrochemical methods, Raman spectroscopy, pulse field gradient (PFG) and electrophoretic NMR (eNMR) techniques, as well as ab initio calculations. The conductivity of the gel containing $10 \mathrm{wt} \%$ polymer is only reduced by $14 \%$ relative to the liquid electrolyte. In addition, the cosolvation by polymer functional groups, a priori expected to slow lithium transport relatively to the anion, has instead a positive effect on lithium transport. Indeed, the ester groups not only participate in lithium solvation and increase ionic dissociation, but since this interaction is rather weak, rather than lowering the lithium diffusion relatively to other species, it mainly decorrelates lithium transport from anionic mobility. Compared to its liquid fraction, the gels show, at the same time, better dissociation and a higher lithium transference number, which results in a higher cationic conductivity, despite the overall conductivity loss.

Received 30th July 2019

Accepted 20th August 2019

DOI: 10.1039/c9ra05917b

rsc.li/rsc-advances be either advantageous or disadvantageous for ionic mobility. Several reports suggest that strong lithium ion coordination by polymer networks containing ether oxygen units (e.g. PEO) participate to low ionic mobility. ${ }^{6-8}$ On the other hand, Tominaga et al. and Brandell et al. ${ }^{7,9}$ reported on loose coordination for polyesters and polycarbonates. Therefore, the polyester PMMA matrix was chosen in this study as it contains $\mathrm{C}=\mathrm{O}$ functional groups, which could be favorable in terms of lithium ionic mobility. In addition, PMMA was reported to exhibit high ionic conductivity as a result of high electrolyte uptake. ${ }^{10,11}$ Besides, from a practical point of view, it is non-toxic and nonfluorinated and PMMA-based gel electrolytes can be easily prepared in situ from monomer and crosslinker low viscosity mixtures and allow long term cycling of lithium-ion batteries. ${ }^{12}$

To investigate ion solvation and coordination in electrolytes, spectroscopic techniques such as RAMAN are commonly used. $^{13,14}$ On the other hand, to investigate ion dynamics, nuclear magnetic resonance (NMR) techniques are useful. With multinuclear pulsed-field-gradient (PFG) NMR, diffusion coefficients of the different species are obtained, which has been widely used to characterize both liquid and polymer electrolytes. ${ }^{15}$ In particular, lithium transference numbers can be estimated, however, only under the assumption of validity of the Nernst-Einstein equation, which is often not fulfilled due to ion pairing. Complementarily to this approach, the model-free study of correlations and coordination behavior in electrolytes by electrophoretic NMR (eNMR) ${ }^{16,17}$ allows the direct calculation of transference numbers from electrophoretic mobilities
${ }^{a}$ Helmholtz Institute Münster, Forschungszentrum Jülich (IEK-12), Corrensstr. 46, 48149 Münster, Germany. E-mail: d.diddens@fz-juelich.de; e.paillard@fz-juelich.de ${ }^{b}$ Institute of Physical Chemistry, University of Münster, Corrensstr. 28/30, 48149 Münster, Germany. E-mail: schonhoff@uni-muenster.de

$\uparrow$ Electronic supplementary information (ESI) available. See DOI: 10.1039/c9ra05917b 
without further assumptions, yielding information about correlated ion motion in an electric field. In recent years, it has been applied to several liquid electrolytes to study ion pairing and transference numbers. ${ }^{17-20}$ For example, in ionic liquids, $\mathrm{Li}-$ anion clusters could be identified as transport vehicles for $\mathrm{Li}^{20,21}$ Provided that mobilities are not too low, eNMR is even applicable to polymer electrolytes to determine transference numbers, which has recently been shown for $\mathrm{Li}$ salt in poly(ethyleneoxide). ${ }^{22}$

In addition to experimental measurements, molecular modelling techniques are extremely helpful to gain insights at the microscopic level. Molecular dynamics (MD) simulations are frequently employed to study ion coordination ${ }^{23-26}$ or ion transport mechanism ${ }^{27-33}$ in electrolyte materials, however, these calculations depend on reliable force fields $s^{34-36}$ to obtain accurate results. On the other hand, ab initio quantum chemistry (QC) calculations require no further input than the molecular structure, and offer access to binding energies between different molecular species. In case of battery materials, several studies focused on the stability of the lithium coordination sphere. ${ }^{37-40}$

Here we study the influence of polymer network addition on electrolyte transport properties in a crosslinked poly(methyl methacrylate) (PMMA) gel polymer electrolyte (PGPE). PGPE allows the cycling of graphite/ $\mathrm{LiNi}_{0.33} \mathrm{Mn}_{0.33} \mathrm{Co}_{0.33} \mathrm{O}_{2}$ (NMC111) lithium ion cells after in situ crosslinking. ${ }^{12}$ Since the investigated gel polymer membrane contains $\mathrm{C}=\mathrm{O}$ groups on the backbone and $-\mathrm{CH}_{2}-\mathrm{CH}_{2}-\mathrm{O}$ - groups (EO) on the oligoethylene oxide crosslinker, these polar groups likely form complexes with lithium salts and organic solvents and consequently increase the salt dissociation degree within the PGPE compared to the corresponding liquid electrolyte. This increased dissociation is investigated here by Raman spectroscopy and by the changes in self-diffusion coefficients and electrophoretic mobilities of the different ion species via ${ }^{7} \mathrm{Li},{ }^{19} \mathrm{~F}$ and ${ }^{13} \mathrm{C}$ PFG and electrophoretic NMR. In addition, we used both density functional theory (DFT) and higher-level QC calculations to characterize the $\mathrm{Li}^{+}$ coordination environment in the microscopically heterogeneous PGPE.

\section{Experimental}

\subsection{Chemicals}

Lithium bis(trifluoromethanesulfonyl)imide (LiTFSI) (BASF) and lithium bis(oxalato)borate (LiBOB) (BASF) salts were dried under reduced pressure of $1 \times 10^{-3} \mathrm{mbar}$ for 2 days at $120^{\circ} \mathrm{C}$ and $110{ }^{\circ} \mathrm{C}$ respectively, while ethylene carbonate (EC) and propylene carbonate (PC) (both BASF, SelectilyteTM) and additives (vinylene carbonate (VC) (BASF, SelectilyteTM) and 1propene-1,3-sultone (PES) (>98\%, TCI Chemicals)) were used as received. Impurity content and water level were analysed by coulometric Karl-Fischer titration (Metrohm 851 Titrando) and liquid ${ }^{1} \mathrm{H},{ }^{1} \mathrm{~F},{ }^{13} \mathrm{C}-\mathrm{NMR}$ (Bruker AVANCE, $300 \mathrm{MHz}$ or $400 \mathrm{MHz}$ ). Methyl methacrylate (MMA) (99\%, Merck) and poly(ethylene glycol) dimethacrylate $\left(M_{\mathrm{n}}=750 \mathrm{~g} \mathrm{~mol}{ }^{-1}\right.$, Merck) were stored on molecular sieve ( $3 \AA$ ) for one week before use. Azobisisobutyronitrile (AIBN, 98\%, Merck) was used as received.

\subsection{PGPE and liquid electrolyte preparation}

All preparation steps for PGPE were carried out in a dry room (dew point $<-65^{\circ} \mathrm{C}$ and $\mathrm{H}_{2} \mathrm{O}<5.3 \mathrm{ppm}$ ). Crosslinking was used for obtaining PGPEs with sufficient mechanical stability at low polymer content. The investigated PGPE contain the polymer precursor and Li salts and solvents which are combined with the ratio of $10: 90 \mathrm{wt} \%$ (polymer precursor: liquid fraction). The proposed polymer precursor contains $95.05 \mathrm{wt} \%$ of MMA monomer, 4.75 wt\% poly(ethylene glycol) dimethacrylate ( $5 \mathrm{wt} \%$ of MMA) and $0.2 \mathrm{wt} \%$ AIBN as thermal initiator, and the corresponding structure is indicated in Fig. 1. Moreover, the liquid fraction was prepared in an Ar-filled glove box $(<0.1 \mathrm{ppm}$ $\mathrm{O}_{2}$ and $\mathrm{H}_{2} \mathrm{O}$ ) and with the formulation of $0.9 \mathrm{M} \mathrm{LiTFSI}+0.1 \mathrm{M}$ LiBOB in EC/PC $7: 3 \mathrm{wt} \%+3 \mathrm{wt} \% \mathrm{VC}+3 \mathrm{wt} \%$ PES. The gel polymer precursor was cast in a frame to control the thickness of the membrane. As the final step, the frame containing the gel precursor was kept in an oven at $75{ }^{\circ} \mathrm{C}$ overnight.

\subsection{Electrochemical testing}

The ionic conductivity of PGPEs was measured using electrochemical impedance spectroscopy (EIS). EIS spectra were acquired on a Novocontrol Alpha-A analyser, equipped with a ZG2 extension interface and a cryostat for temperature control in the frequency range from $1 \mathrm{MHz}$ to $0.1 \mathrm{~Hz}$ and in the temperature range from -30 to $80{ }^{\circ} \mathrm{C}$ with $10{ }^{\circ} \mathrm{C}$ intervals and temperature equilibration for $30 \mathrm{~min}$ prior to each measurement. PGPEs were sandwiched between two stainless steel electrodes with $100 \mu \mathrm{m}$ gap (using a $14 \mathrm{~mm}$ inner diameter PTFE spacer in a 2032 coin cell). Liquid electrolyte ionic conductivity was measured with a MCS10 EIS-based multichannel conductivity meter from Bio-logic with the same temperature range and interval as for PGPEs.

\subsection{Thermal analysis}

Differential scanning calorimetry (DSC) was conducted using a DSC Q2000 (TA instruments) to investigate glass transition temperatures. All the samples were hermetically sealed in

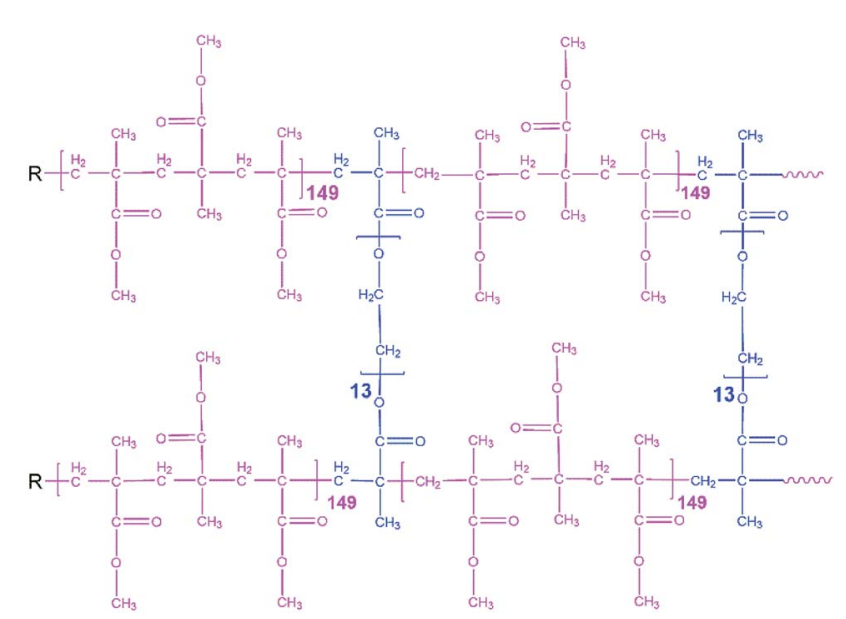

Fig. 1 PGPE chemical structure, $\mathrm{C}=\mathrm{O}$ groups on backbone and $\mathrm{EO}$ groups on crosslinker. 
aluminum TzeroTM pans in a dry room atmosphere (dew point $<-65{ }^{\circ} \mathrm{C}$ and $\mathrm{H}_{2} \mathrm{O}<5.3 \mathrm{ppm}$ ). The samples were cooled from 20 to $-150{ }^{\circ} \mathrm{C}$ and heated to $150{ }^{\circ} \mathrm{C}$ for PGPE and $120{ }^{\circ} \mathrm{C}$ for liquid fraction, respectively, at $10 \mathrm{~K} \mathrm{~min}^{-1}$ for three cycles under $\mathrm{He}$ flow.

\subsection{Spectroscopic analysis}

2.5.1 Raman measurements. Raman measurements were conducted on a Bruker VERTEX 70 spectrometer at $25{ }^{\circ} \mathrm{C}$ equipped with HFS600E-PB4 temperature stage and T95LinkPad controller. Raman spectroscopy was carried out with a laser source at $9395 \mathrm{~cm}^{-1}$ and with a power of $500 \mathrm{~mW}$. The spectra resolution was $2 \mathrm{~cm}^{-1}$ in the range from $0 \mathrm{~cm}^{-1}$ to $4000 \mathrm{~cm}^{-1}$.

2.5.2 PFG-NMR measurements. All NMR experiments were performed on a Bruker AVANCE III HD 400 spectrometer with a gradient probe head (Diff50, Bruker) and selective radiofrequency inserts for ${ }^{7} \mathrm{Li},{ }^{19} \mathrm{~F}$ and ${ }^{13} \mathrm{C}$. The maximum gradient strength was $2800 \mathrm{G} \mathrm{cm}^{-1}$. The temperature was controlled using a GMH 3710 controller with a PT100 thermocouple (Greisinger electronics, Germany).

The self-diffusion coefficient $D$ for each species was measured with a stimulated echo pulse sequence in a pulsedfield-gradient NMR (PFG-NMR) experiment. $D$ was calculated by using eqn (1) with the gyromagnetic ratio $\gamma$, the gradient strength $g$, the gradient duration $\delta(1 \mathrm{~ms})$ and the observation time $\Delta(100 \mathrm{~ms})$.

$$
I(g)=I(0) \exp \left(-\gamma^{2} g^{2} \delta^{2} D\left(\Delta-\frac{\delta}{3}\right)\right)
$$

2.5.3 eNMR measurements. eNMR experiments were performed using a previously described ${ }^{\mathbf{1 7}}$ self-built electrode configuration fitting in a $5 \mathrm{~mm}$ NMR tube and a self-built pulse generator $\left(U_{\max }=1 \mathrm{kV}, I_{\max }=50 \mathrm{~mA}\right)$ to apply the electric field. By applying a voltage $U$ during a double stimulated echo pulse sequence, a phase shift of the spectrum could be observed. By increasing $U$ in a series of spectra, one can extract the electrophoretic mobility $\mu$ from the slope in a linear plot of $\Delta \Phi$ against $U$ by using eqn (2) with the known parameters $\gamma, g, \delta(1 \mathrm{~ms})$, $\Delta_{\mathrm{drift}}(100 \mathrm{~ms})$ and the electrode distance $d(22 \mathrm{~mm})$.

$$
\Delta \Phi=\gamma g \delta \Delta_{\text {drift }} \frac{U}{d} \mu
$$

The gradient strength $g$ varied between $100 \mathrm{G} \mathrm{cm}^{-1}$ and 500 $\mathrm{G} \mathrm{cm}^{-1}$. $U$ was increased in 21 steps from $0 \mathrm{~V}$ to $120 \mathrm{~V}$ with alternating sign of the voltage to cover the largest possible voltage range without decomposition of the sample. The phase shift analysis was performed by fits of the spectra with phase sensitive Lorentzian profiles as described elsewhere. ${ }^{41}$

\subsection{Quantum chemical calculations}

All QC calculations have been performed with the Gaussian 16 package. ${ }^{42}$ In a first step, the binding energies and free energies between a lithium ion and a single EC, PC, TFSI, or BOB molecule, as well as an MMA monomer and dimer (denoted as $(\mathrm{MMA})_{2}$ ) have been computed. Additionally, the interaction of $\mathrm{Li}^{+}$with tetraethylene glycol dimethyl ether (TEGDME) has been computed as a model for lithium ions coordinating to the longer oligoethylene oxide cross-linkers. In a second step, we performed calculations of larger clusters composed of the aforementioned molecules mimicking the entire lithium solvation shell in the electrolyte..$^{38-40}$

For the $\mathrm{Li}^{+}$-solvent, $\mathrm{Li}^{+}$-anion and $\mathrm{Li}^{+}$-dimers, we compared computationally expedient $\mathrm{PBE} / 6-31+\mathrm{G}(\mathrm{d}, \mathrm{p})$ calculations to higher-level calculations with the accurate but computationally expensive G4MP2 composite method. ${ }^{43}$ Furthermore, for the PBE/6-31+G(d,p) calculations, we also assessed the effect of Grimme's D3 dispersion correction with Becke-Johnson damping $^{44}$ on the results. Solvent effects were taken into account via the implicit SMD model ${ }^{45}$ using a dielectric constant of $\varepsilon=20$ characteristic for carbonate mixtures. ${ }^{38}$ For TFSI, both mono- and bidentate coordinations have been taken into account. For BOB, we disregarded a possible coordination via the oxygen atoms linked to the central boron atom, and only took coordination via the double bonded oxygen into account. The results for the dimer calculations are shown in Table S1 in the ESI. $\dagger$

As reported previously, ${ }^{38-40}$ the $\mathrm{PBE}$ calculations reproduce the G4MP2 values surprisingly well for EC, PC, PMMA, and BOB. Here, the deviations are mainly even below $1 \mathrm{kcal} \mathrm{mol}^{-1}$, which is comparable to the intrinsic uncertainty of G4MP2. ${ }^{43}$ For TEGDME and monodentate TFSI, however, the deviations are $4 \mathrm{kcal} \mathrm{mol}^{-1}$ and $2 \mathrm{kcal} \mathrm{mol}^{-1}$, respectively. For the calculations of larger clusters involving TEGDME or monodentate TFSI (see below), we therefore used the (free) energy difference between the PBE and the G4MP2 calculations for the $\mathrm{Li}^{+}$-TEGDME or monodendate $\mathrm{Li}^{+}$-TFSI dimer as a correction to the cluster energy as motivated previously. ${ }^{39,40}$ We also note that the inclusion of an empirical dispersion correction $^{\mathbf{4 4}}$ did not improve the accuracy of the PBE/6-31+G(d,p) calculations, but rather led to larger deviations from the G4MP2 reference calculations (Table $\mathrm{S} 1 \dagger$ ). Therefore, we abstained from using a dispersion correction for the following cluster calculations.

Motivated by the good agreement between PBE/6-31+G(d,p) and G4MP2 calculations, we finally performed calculations of larger clusters at the $\mathrm{PBE} / 6-31+\mathrm{G}(\mathrm{d}, \mathrm{p})$ level, in which the lithium ion is surround by a full solvation shell with different compositions (see Fig. S1 in $\mathrm{ESI}_{\dagger}^{\dagger}$ ). In all calculations except those involving TEGDME, we fixed the coordination number of $\mathrm{Li}^{+}$to four. However, since PMMA, TEGDME, TFSI, or BOB may coordinate via multiple sites, some of the four coordination sites may originate from the same molecule. In addition to the explicit modelling of the first coordination shell, we modelled solvation effects beyond the first coordination shell by the SMD model $^{45}$ as described above.

\section{Results and discussion}

\subsection{Ionic conductivities and thermal analysis}

According to eqn (3), ionic conductivity is dependent on ionic mobility $\left(\mu_{\mathrm{i}}\right)$ and on the number density of ions $\left(n_{\mathrm{i}}\right)$ for each ion 
species i. As can be seen in Fig. 2(a), the ionic conductivity of the EC/PC liquid electrolyte is $4.5 \mathrm{mS} \mathrm{cm}^{-1}$. The PGPE with $10 \mathrm{wt} \%$ polymer exhibits a reduced ionic conductivity of $3.9 \mathrm{mS} \mathrm{cm}^{-1}$ at $20{ }^{\circ} \mathrm{C}$, presumably due to viscosity increase, geometrical restriction $^{46}$ and interactions between ions and polymer chain. The conductivities are, however, relatively close to each other, with the gel value reaching $86 \%$ of that of the liquid fraction.

$$
\sigma=\sum_{\mathrm{i}} n_{\mathrm{i}} \mu_{\mathrm{i}} z_{\mathrm{i}} e
$$

Moreover, as seen in Fig. 2(b) the PGPE exhibits a low $T_{\mathrm{g}}$, similar to that of the liquid fraction $\left(-89.5{ }^{\circ} \mathrm{C}, v s .-89.9^{\circ} \mathrm{C}\right.$ at the inflexion point at $10 \mathrm{~K} \mathrm{~min}^{-1}$ ), which indicates that the presence of the polymer hardly affects the average mobility of the electrolyte species compared to the liquid electrolyte. No crystallization is observed on the cooling ramps and cold crystallization and melting are only slightly affected by the presence of the polymer (the presence of the polymer offsets the cold crystallization temperature onset) and the peaks are small in both cases. The presence of the polymer matrix slightly

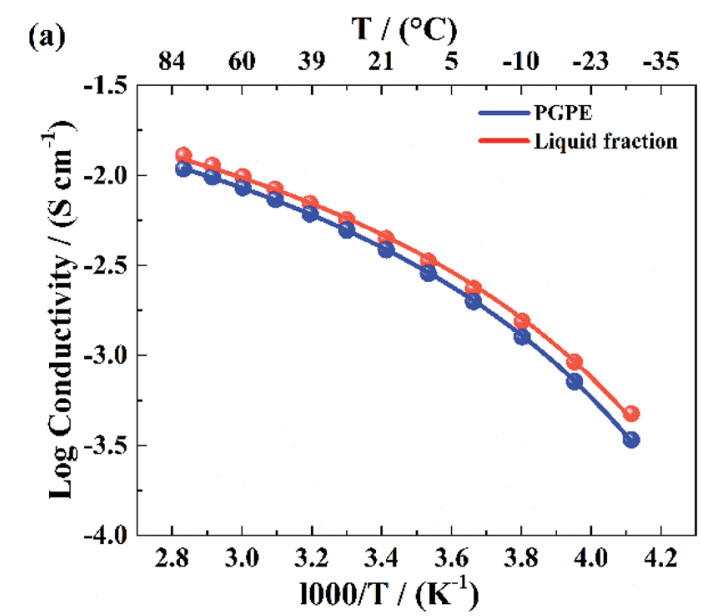

(b)

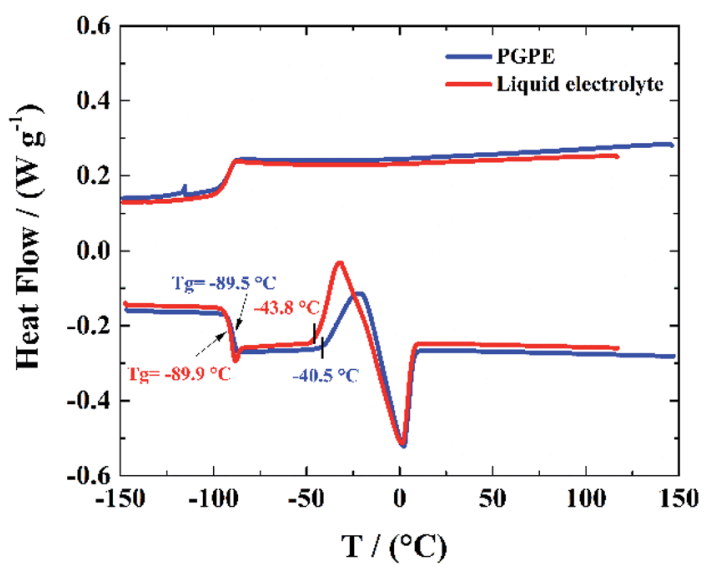

Fig. 2 (a) Ionic conductivity of PGPE and liquid electrolyte. The data points are fitted by VTF function. (b) DSC thermogram, lower curves: first heating ramp and upper curves: cooling trace, both of $10 \mathrm{~K} \mathrm{~min}^{-1}$ for PGPE (blue) and liquid electrolyte (red). decreases the cold crystallization and melting enthalpies ( $c a .16$ $\mathrm{J} \mathrm{g}^{-1}$ vs. $21 \mathrm{~J} \mathrm{~g}^{-1}$ ), either due to lowering the crystal fraction or lowering its melting enthalpy.

\subsection{Degree of ion dissociation}

Raman spectroscopy was performed to provide complementary insights concerning the dissociation degree of the Li salts in the gel polymer electrolyte.

The Raman spectra of the EC/PC-based liquid electrolyte and the PGPE are shown in Fig. 3(a) and (b) respectively in the 690$760 \mathrm{~cm}^{-1}$ range. They exhibit two peaks: one at $719 \mathrm{~cm}^{-1}$, corresponding to the symmetric ring deformation of $\mathrm{EC}^{47}$ and one at $743 \mathrm{~cm}^{-1}$, corresponding to the $\mathrm{S}-\mathrm{N}-\mathrm{S}$ stretching mode of the TFSI anion. This peak is sensitive to TFSI solvation and can be split into two contributions: one a at $739 \mathrm{~cm}^{-1}$, corresponding to free TFSI anions and one at $744 \mathrm{~cm}^{-1}$, attributed to contact ion pair (CIP).$^{48}$ Thus, the spectra were fitted with pseudo-Voigt functions to determine the fractions of TFSI in each solvating state. The gel and liquid electrolytes studied here contain relatively dilute salt solutions ( $1 \mathrm{M}$ ) and a well dissociating solvent mixture and are thus expected to contain significant fractions of free anions. ${ }^{48}$ Indeed, the fractions of free anion are high in both cases and is even higher in the case of PGPE (83\%)
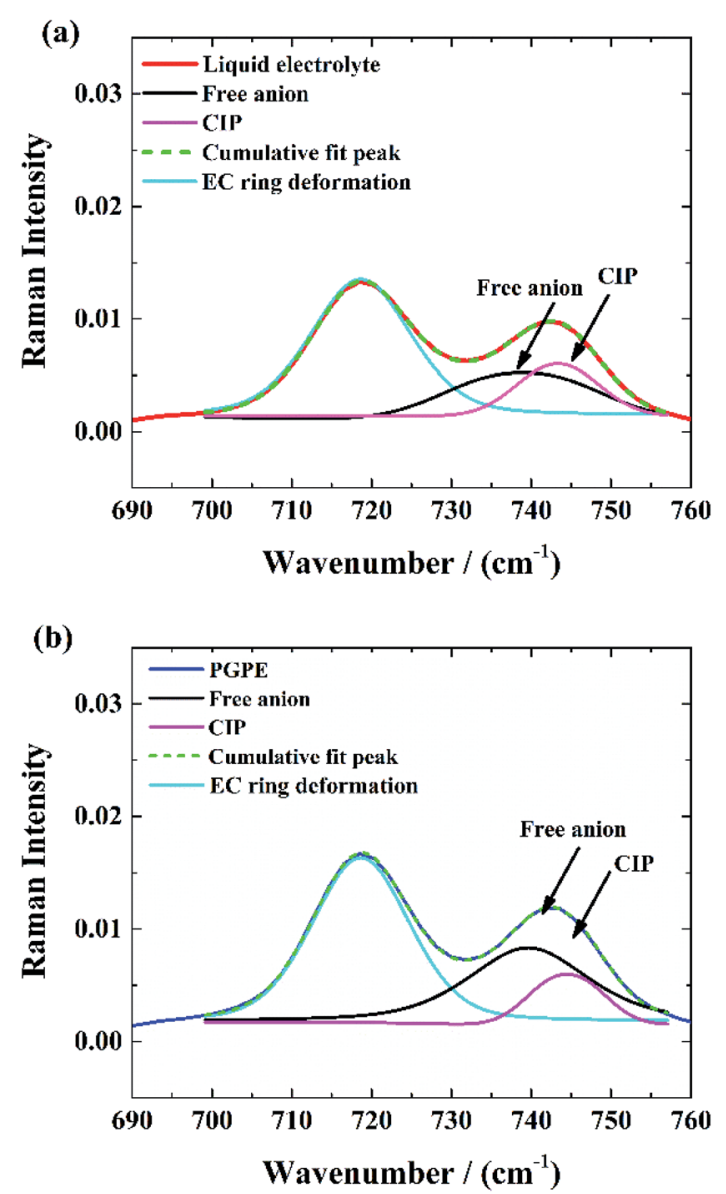

Fig. 3 Deconvolution of $\mathrm{S}-\mathrm{N}-\mathrm{S}$ stretching vibrational mode of the TFSI-anion: (a) in liquid electrolyte, (b) in PGPE. 
compared to the liquid electrolyte (71\%) which indicates a higher degree of salt dissociation in gel polymer electrolytes.

\subsection{Diffusivities}

To further validate the dissociation degree calculated from Raman spectroscopy measurements, self-diffusion coefficients of all involved ionic species were determined via PFG-NMR measurements. Fig. 4 shows the data obtained for the PGPE and the liquid electrolyte for three observed nuclei $\left({ }^{19} \mathrm{~F},{ }^{7} \mathrm{Li}\right.$, ${ }^{13} \mathrm{C}$ ). The spectral lines that were evaluated correspond to $\mathrm{Li}$, TFSI and BOB, respectively. We note here that NMR quantities measured over an observation time in the order of $100 \mathrm{~ms}$ in liquids present fast exchange averages over different species. This implies for example that Li diffusion coefficients (and also mobilities) are average values over free $\mathrm{Li}^{+}$, undissociated $\mathrm{Li}$ ion pairs and any further clusters. In Fig. 4, it is evident that $D^{7} \mathrm{Li}$ and $D^{19} \mathrm{~F}$ are strongly decreasing from the liquid electrolyte to the PGPE. Within the margin of error $D^{13} \mathrm{C}$ is not changing at all. A certain decrease of the diffusion coefficients is expected due to the increase in viscosity and the geometrical restriction arising from polymer addition to the liquid electrolyte. ${ }^{46}$ However, the behavior of $D^{13} \mathrm{C}$ is rather unexpected and suggests that the effect of geometrical restriction is compensated by other factors. Such factors may be changes in ion coordination and clustering, which can partly compensate for the geometrical restriction, as recently shown in similar polymer gel electrolyte systems. $^{46}$

An average salt dissociation degree $\alpha$ can be estimated from the diffusion data in combination with conductivities. An estimate for $\alpha$ (eqn (4)) is given by the ratio of the conductivity measured by impedance spectroscopy $\sigma_{\text {imp }}$ and the conductivity calculated via the Nernst-Einstein equation $\sigma_{\mathrm{PFG}}$ (eqn (5)).

$$
\begin{gathered}
\alpha=\frac{\sigma_{\mathrm{imp}}}{\sigma_{\mathrm{PFG}}} \\
\sigma_{\mathrm{PFG}}=\frac{F^{2}}{R T} \sum c_{\mathrm{i}} z_{\mathrm{i}} D_{\mathrm{i}}
\end{gathered}
$$

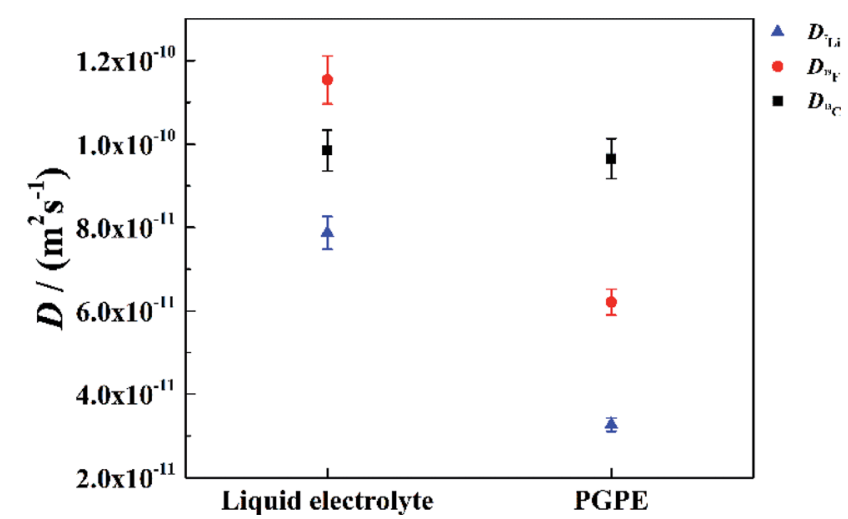

Fig. 4 Self-diffusion coefficients of PGPE and liquid electrolyte: Li (blue triangles), TFSA (red circles) and BOB (black squares) at $25^{\circ} \mathrm{C}$ measured by ${ }^{7} \mathrm{Li},{ }^{19} \mathrm{~F}$ and ${ }^{13} \mathrm{C}$ PFG-NMR, respectively. Errors are calculated by error propagation.
In Table 1 the values for $\alpha$ are shown and compared to those extracted from the Raman spectra. For both systems, $\alpha$ is in very good agreement with the values derived from Raman spectra. A dissociation degree of $81 \%$ in the case of the PGPE shows, in comparison to $67 \%$ for the liquid electrolyte, a significant increase in dissociation. This underlines the positive effect of the gel on ion dissociation as previously shown in the literature. ${ }^{49,50}$

\subsection{Lithium coordination by polar groups of polymer}

The $\mathrm{C}=\mathrm{O}$ stretching mode of $\mathrm{PMMA}^{7,51,52}$ is also convenient to investigate lithium coordination. Fig. 5 shows the Raman spectra of the neat PGPE precursor solution without salt or liquid electrolyte (black curve), with the $\mathrm{C}=\mathrm{O}$ stretching band of the MMA monomer at $1730 \mathrm{~cm}^{-1}$. For the PGPE without any solvents (pink curve), this band evolves toward lower frequencies and adopts a more complex shape, as a result of the $\mathrm{Li}^{+}$ solvation by the 'dry' polymer. The presence of alkyl carbonate solvents (EC and PC) in the PGPE (blue curve), leads to the appearance of a peak at $1760-1840 \mathrm{~cm}^{-1}$ which is assigned to the $\mathrm{Li}^{+}$solvation by the $\mathrm{C}=\mathrm{O}$ groups in EC/PC (stretching band of $\mathrm{C}=\mathrm{O}$ ). However, a small peak remains around $1730 \mathrm{~cm}^{-1}$ and confirms the co-solvation by the polymer in the presence of solvents. The dominance of the Li coordination to the carbonates also indicates that the transport mechanism should be dominated by organic solvents rather than polymer network. ${ }^{7}$

\subsection{Electrophoretic mobility}

For a deeper look into correlated ion motion in the investigated system, electrophoretic NMR (eNMR) experiments are very useful. In contrast to PFG-NMR, with eNMR it is possible to obtain directly the electrophoretic mobilities of all species containing NMR-active nuclei without assuming ideal, noncorrelated ion motion. Unfortunately, due to lower signal intensity, it was not possible to obtain electrophoretic mobilities of the ${ }^{13} \mathrm{C}$ nucleus and therefore of the $\mathrm{BOB}$ anion. However, the electrophoretic mobilities of the lithium $\left({ }^{7} \mathrm{Li}\right)$ and TFSI $\left({ }^{19} \mathrm{~F}\right)$ species could be determined. At this point it should be mentioned, that the observation time in the measurement (100 ms) is much larger than the lifetime of a distinguishable lithium-anion cluster. Therefore, all mobilities are weighted average values over single ions and all existing ion pairs or clusters. The mobilities $\mu^{{ }_{19} \mathrm{~F}}$ and $\mu^{{ }^{7} \mathrm{Li}}$ are shown in Fig. 6 together with the apparent mobilities, which were calculated via the

Table 1 Dissociation degree $\alpha=\left(\frac{\sigma_{\mathrm{imp}}}{\sigma_{\mathrm{PFG}}}\right)$ of PGPE and liquid electrolyte calculated from impedance and PFG-NMR measurements, respectively

\begin{tabular}{lll}
\hline & \multicolumn{2}{c}{ Dissociation degree $\alpha$} \\
\cline { 2 - 3 } Sample & Raman & PFG-NMR \\
\hline Liquid electrolyte & $71 \%$ & $67 \%$ \\
PGPE & $83 \%$ & $81 \%$
\end{tabular}




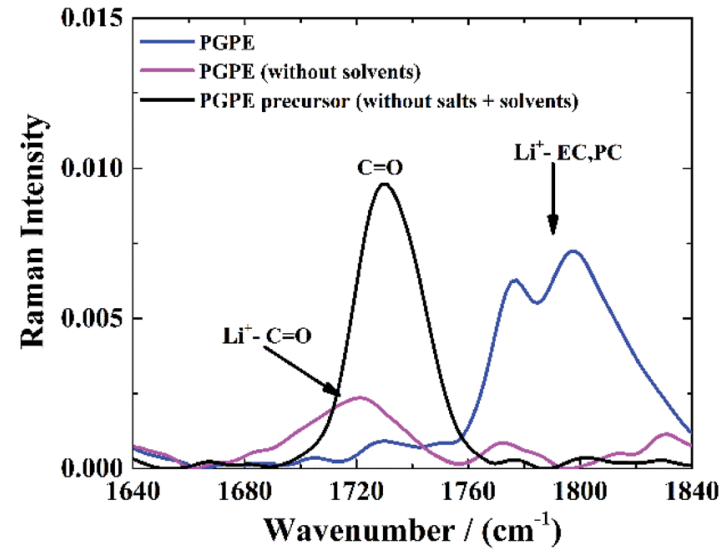

Fig. 5 Raman spectra of $\mathrm{C}=\mathrm{O}$ stretching mode of PMMA by introducing the same salts and solvents as in PGPE.

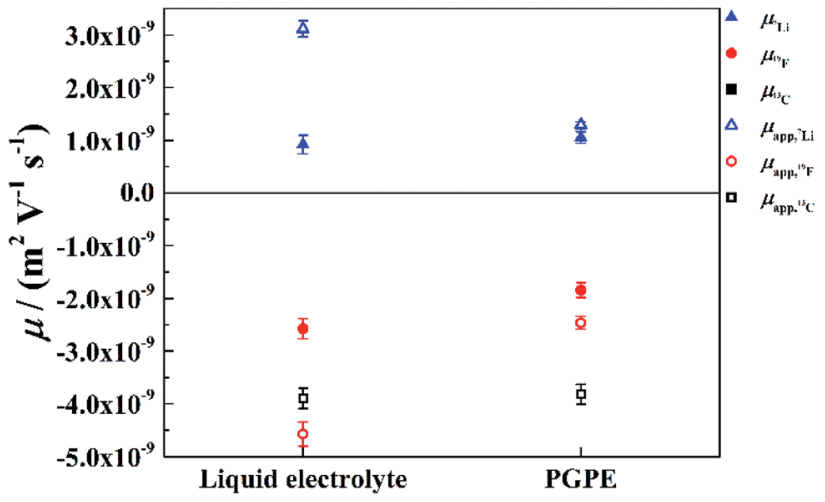

Fig. 6 Electrophoretic mobilities (filled symbols) from eNMR and apparent mobilities (open symbols) from PFG-NMR of the GPE and the liquid electrolyte: Li (blue triangles), TFSA (red circles) and BoB (black squares) at $20{ }^{\circ} \mathrm{C}$ measured by ${ }^{7} \mathrm{Li},{ }^{19} \mathrm{~F}$ and ${ }^{13} \mathrm{C}$ NMR respectively. Errors are calculated by error propagation.

Nernst-Einstein equation assuming completely uncorrelated ion motion.

In the liquid electrolyte, the apparent mobilities deviate strongly from the values measured by eNMR, which shows that the former are heavily affected by ion correlations. It is immediately evident that these correlations are decreased by the presence of the polymer, as the apparent values provide better approximations to the electrophoretic mobilities in the case of the gel as compared to the liquid electrolyte (Fig. 6). Thus, our conclusion is that the gel promotes the disintegration of ion clusters. It is interesting, however, that the mobilities $\mu_{\mathrm{Li}}$ and $\mu_{19} \mathrm{~F}$ are affected by the polymer in a slightly different way: the crosslinked polymer reduces the electrophoretic mobility of TFSI, while in contrast, $\mu^{7} \mathrm{Li}$ has the same value within the margin of error for both electrolytes. This asymmetry in the behavior of the Li cation and the anion cannot be explained by a simple enhancement of salt dissociation, i.e. the lowering of the ion pair fraction, and will be discussed in the next paragraph.

Further light is shed on ion speciation by the concept of the effective charge $\varepsilon_{\mathfrak{c}}^{i}($ eqn $(6)),{ }^{17}$ which relates the average charge of a species containing the nucleus in question to the nominal charge of the uncorrelated single ion. ${ }^{17}$ Values below $\varepsilon_{\mathrm{c}}^{\mathrm{i}}=1$ thus indicate deviations from ideal uncorrelated behavior.

$$
\varepsilon_{\mathrm{c}}^{\mathrm{i}}=\frac{\mu_{\mathrm{i}}}{\mu_{\mathrm{app}, \mathrm{i}}}
$$

In Fig. 7 the effective charges of the TFSI and the lithium species are shown. Both increase due to polymer addition, reflecting again the reduction of ion correlations in the gel. $\varepsilon_{\mathrm{c}} \mathrm{F}$ rises from 0.56 in the liquid electrolyte up to 0.75 in the PGPE, however, $\varepsilon_{\mathrm{c}}{ }^{19} \mathrm{Li}$ rises by a greater extent from 0.30 up to 0.82 . If assuming only Li-TFSI and Li-BOB ion pairs in equilibrium with single ions, this difference cannot be explained, since then ion pair dissociation would be symmetric, leading to identical effective charges of cation and anion. We rather have to conclude on the presence of larger, asymmetric ion clusters dominating charge transport. Similar arguments based on differing effective charges of cation and anions were recently given for ionic liquids. ${ }^{17}$ It seems that, even in the dilute carbonate-based electrolyte, asymmetric clusters are more relevant than ion pairs. For such clusters, the polymer addition reduces correlations of $\mathrm{Li}$ to a considerably higher extent than those of TFSI. Note that in the PGPE, the effective charges of Li and anion are similar, indicating a shift from asymmetric to symmetric clusters. These results demonstrate the positive effect of the polymer matrix on the dissociation of the system in general, in particular mobilizing the lithium cation.

\subsection{Transference number}

Regarding a possible application of the investigated electrolyte system, the lithium transference number $t^{7} \mathrm{Li}$ is an important quantity to estimate the performance of the electrolyte considering the lithium ion motion. In Table 2, the lithium transference number $t^{7} \mathrm{Li}$ calculated from eNMR data is shown. In the PGPE the lithium transference number of 0.34 is considerably higher than in the liquid electrolyte $\left(t_{\overline{L i}}=0.25\right)$ and the $\mathrm{Li}^{+}$ ionic conductivity $\left(t_{\mathrm{Li}} \times \sigma\right)$ increases from $1.13 \mathrm{mS} \mathrm{cm}^{-1}$ for the

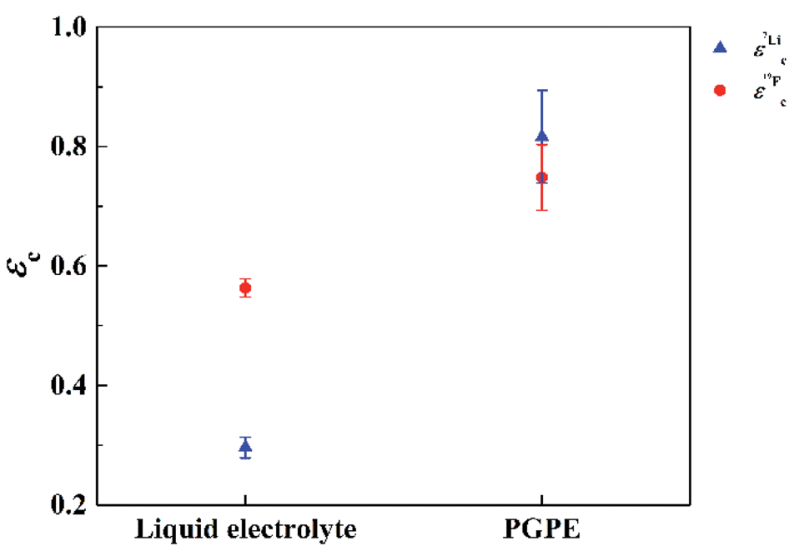

Fig. 7 Effective charges for Li (blue triangles) and TFSI (red circles) at $20^{\circ} \mathrm{C}$ measured by ${ }^{7} \mathrm{Li}$ and ${ }^{19} \mathrm{~F}$ NMR respectively. Errors are calculated by error propagation. 
Table 2 Transference number $t_{\mathrm{Li}}$ calculated from electrophoretic mobilities measured at $20{ }^{\circ} \mathrm{C}$ for the liquid electrolyte and PGPE. Apparent transference number $t_{\mathrm{app},{ }^{\prime} \mathrm{Li}}$ from PFG-NMR are given for comparison. Errors are calculated by error propagation

\begin{tabular}{lll}
\hline Sample & $t^{{ } \mathrm{Li}}$ & $t_{\mathrm{app},}{ }^{7} \mathrm{Li}$ \\
\hline Liquid electrolyte & $0.25 \pm 0.02$ & $0.41 \pm 0.04$ \\
PGPE & $0.34 \pm 0.02$ & $0.33 \pm 0.03$
\end{tabular}

liquid electrolyte to $1.33 \mathrm{mS} \mathrm{cm}^{-1}$ for PGPE. Thus, the beneficial effect of the polymer concerning the reduction of ion correlations in particular for Li shows up very clearly in the transference number. The last column gives a comparison to the estimates of $t_{\mathrm{app},{ }^{7} \mathrm{Li}}$ from PFG-NMR. It is interesting that the PGPE shows similar values for $t_{7} \mathrm{Li}$ and $t_{\mathrm{app}},{ }^{7} \mathrm{Li}$, implying that $t_{7} \mathrm{Li}$ has even reached the value which would be expected for an ideal system without ion correlations.

\subsection{Clusters stabilities based on DFT calculation}

Finally, we employ QC calculations of fully solvated $\mathrm{Li}^{+}$clusters to further rationalize the solution structure within the liquid electrolyte and the PGPE, similar in spirit to recent studies on related electrolytes. ${ }^{38-40}$ Fig. 8 shows the formation energies $\Delta E$ and free energies $\Delta G$ for various lithium ion clusters comprising the individual electrolyte components (i.e. solvent, polymer and anions). As a reference, we chose the $\left[\operatorname{Li}(\mathrm{EC})_{4}\right]^{+}$ cluster modelling a lithium ion that is fully solvated in the liquid phase of the electrolyte. The snapshots of the corresponding clusters in Fig. 8 are shown in Fig. S1 in the ESI, $\uparrow$ and the precise values of the energies presented in Fig. 8 are shown in Table S2. $\dagger$ First, we note that the coordination to TEGDME a proxy for the PEO cross-linker - shows negative values for $\Delta E$ and highly negative values for $\Delta G$ for both the

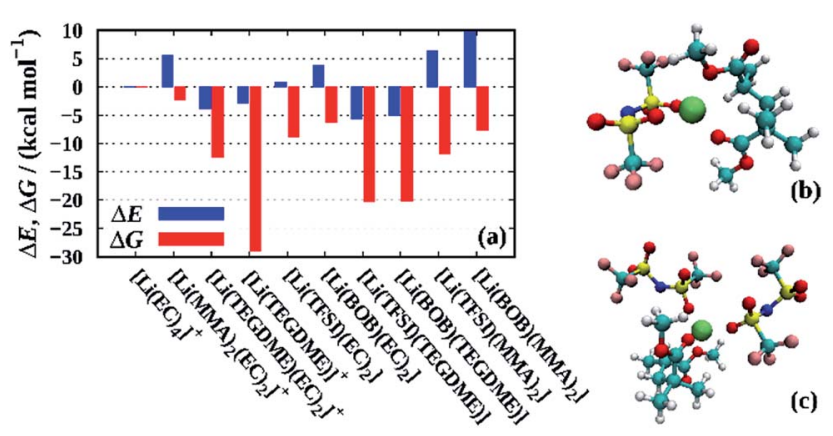

Fig. 8 (a) Formation energies $\Delta E$ and free energies $\Delta G$ for various lithium ion clusters calculated at the PBE/6-31+G(d,p) level of theory. In all cases except those involving TEGDME, the total coordination number has been constrained to four, while for TEGDME also higher coordination numbers as determined from the geometry optimization (see Fig. $\mathrm{S} 1 \dagger$ ) have been taken into account. For clusters involving TEGDME, a correction based on the energy differences between PBE and G4MP2 calculations has been applied (see Table S1 $\uparrow$ and main text). As a reference, we chose the $\left[\mathrm{Li}(\mathrm{EC})_{4}\right]^{+}$cluster modelling a lithium ion fully solvated by carbonate molecules. Snapshots of the $\left[\mathrm{Li}(\mathrm{TFSI})(\mathrm{MMA})_{2}\right]$ and $\left[\mathrm{Li}(\mathrm{TFSI})_{2}(\mathrm{MMA})_{2}\right]^{-}$clusters that partially immobilize cations and anions are shown in (b) and (c), respectively.
$\left[\mathrm{Li}(\mathrm{TEGDME})(\mathrm{EC})_{2}\right]^{+}$cluster $\left(\mathrm{Li}^{+}\right.$coordination to two ether oxygen atoms) and the $[\mathrm{Li}(\mathrm{TEGDME})]^{+}$cluster (coordination to all TEGDME oxygen atoms). Thus, it implies that the lithium ions readily coordinate to the crosslinking PEO chains in the electrolyte. However, since the crosslinkers are only employed at low mole fractions (see Section 2.2), these sites are likely to be fully coordinated due to the strong interaction with $\mathrm{Li}^{+}$, thus preventing an excessive binding of a large fraction of lithium ions to the crosslinkers. The comparison of the respective values for $\Delta E$ and $\Delta G$ demonstrates that the $\mathrm{Li}^{+}$-TEGDME binding is largely stabilized by the entropic gain resulting from the release of four EC molecules from the $\mathrm{Li}^{+}$solvation shell of $\left[\mathrm{Li}(\mathrm{EC})_{4}\right]^{+}$.

A similar effect is found for the formation of contact ion pairs with either TFSI or BOB, modeled by the clusters $\left[\mathrm{Li}(\mathrm{TFSI})(\mathrm{EC})_{2}\right]^{+}$and $\left[\mathrm{Li}(\mathrm{BOB})(\mathrm{EC})_{2}\right]^{+}$. Here, $\Delta E$ is slightly positive in both cases, however, $\Delta G$ is significantly negative, overall rendering both types of ion pairs stable. Contrarily, when TEGDME, instead of EC, accounts for the partial solvation of the ion pairs, i.e. $[\mathrm{Li}(\mathrm{TFSI})(\mathrm{TEGDME})]^{+}$and $[\mathrm{Li}(\mathrm{BOB})(\mathrm{TEGDME})]^{+}$, both $\Delta E$ and $\Delta G$ are negative, which suggests that lithium ions coordinating to the crosslinker are more likely engaged in ion pairs than fully solvated by the liquid electrolyte. We also note that the $\left[\mathrm{Li}(\mathrm{PC})_{4}\right]^{+}$cluster is slightly more stable than the $\left[\mathrm{Li}(\mathrm{EC})_{4}\right]^{+}$cluster, both energetically $\left(\Delta E=-1.5 \mathrm{kcal} \mathrm{mol}^{-1}\right)$ and entropically ( $\Delta G=-1.8 \mathrm{kcal} \mathrm{mol}^{-1}$, not shown). However, since these differences are rather small, we only expect a slight preference of $\mathrm{Li}^{+}$to coordinate to PC.

Interestingly, while the coordination of a single $\mathrm{Li}^{+}$to PMMA (modeled by the coordination to a dimer, i.e. $\left[\operatorname{Li}(\mathrm{MMA})_{2}(\mathrm{EC})_{2}\right]^{+}$) only displays a slightly negative value for $\Delta G$ in the range of a few $\mathrm{kcal} \mathrm{mol}^{-1}$, the corresponding free energies for the anioncontaining PMMA clusters [Li(TFSI)(MMA) $\left.)_{2}\right]$ and $\left[\mathrm{Li}(\mathrm{BOB})(\mathrm{MMA})_{2}\right]$ are substantially negative with $\Delta G$ values of -12 and $-8 \mathrm{kcal} \mathrm{mol}^{-1}$, respectively. As before, this stabilization mainly stems from an entropic gain resulting from the release of the EC molecules (see above). Moreover, comparative calculations of $\mathrm{Li}^{+}$-PMMA clusters containing two anions, that is $\left[\mathrm{Li}(\mathrm{TFSI})_{2}(\mathrm{MMA})_{2}\right]^{-}$and $\left[\mathrm{Li}(\mathrm{BOB})_{2}(\mathrm{MMA})_{2}\right]^{-}$, show values in the range of -1.8 to $4.4 \mathrm{kcal} \mathrm{mol}^{-1}$ and -6.9 to $-2.1 \mathrm{kcal} \mathrm{mol}^{-1}$ for $\Delta E$ and $\Delta G$, respectively (Table $\mathrm{S} 2 \dagger$ ), suggesting that also two distinct anions might indirectly bind to the PMMA chains via a lithium ion. In all cases, this indirect binding to PMMA is more pronounced for TFSI as compared to BOB in agreement with the stronger immobilization of TFSI observed experimentally.

Although the impact of the solution structure on its dynamical properties probed by PFG-NMR or eNMR remain inaccessible in our calculations, our results nonetheless rationalize the eNMR results on a qualitative level, which indicate that, while the mobility of the lithium ions in the gel is approximately the same as in the liquid electrolyte within the uncertainties, the anions (mainly TFSI) are slower in the gel than in the liquid electrolyte (see Section 3.5). Due to the formation of larger ion clusters in the vicinity of the PMMA backbone (and also of the less prevalent crosslinkers), the anions become at least partially immobilized. In addition, the 
large size of the TFSI anions might further hinder their motion through the dense polymer network. Even though the corresponding solvated $\left[\mathrm{Li}(\mathrm{EC})_{4}\right]^{+}$cluster has a comparable size as a TFSI anion, and might thus also be slowed down by the polymer network, the rapid exchange of its solvation shell, however, would still render the lithium ions more mobile. To this end, a future MD simulation study to fully unravel the lithium ion transport mechanism in these electrolytes would be highly promising.

\section{Conclusion}

The good performance in terms of conductivity of PMMA gel polymer electrolytes $v s$. its liquid electrolyte fraction is linked on the one hand, to the well retained mobility of ionic species in combination with a reduced effect of ion correlations on lithium mobility as indicated by eNMR, DSC and Raman spectroscopy. Interestingly, the polymer backbone interacts with the lithium cation and, besides increasing ionic dissociation, it slows down the anion while leaving the mobility of the lithium cation almost unaffected. With an improved effective lithium transference number calculated from eNMR, the loss of conductivity, a priori due to the volume occupation of the immobile polymer matrix is compensated in the PGPE by a $15 \%$ increase in $\mathrm{Li}^{+}$conductivity $\left(\sigma t_{\mathrm{Li}}\right)$ vs. liquid electrolyte (i.e. 1.33 $\mathrm{mS} \mathrm{cm}{ }^{-1}$ vs. $1.13 \mathrm{mS} \mathrm{cm}^{-1}$ at $20{ }^{\circ} \mathrm{C}$ ).

\section{Conflicts of interest}

The authors declare that they have no competing interests.

\section{Acknowledgements}

Part of the research presented here conducted under BenchBatt project funded by the Federal Ministry of Education and Research (BMBF) (grant no. 03XP0047B).

\section{Notes and references}

1 L. Long, S. Wang, M. Xiao and Y. Meng, J. Mater. Chem. A, 2016, 4, 10038-10069.

2 B. Oh, W. I. Jung, D.-W. Kim and H. W. Rhee, Bull. Korean Chem. Soc., 2002, 23, 683-687.

3 H.-D. Nguyen, G.-T. Kim, J. Shi, E. Paillard, P. Judeinstein, S. Lyonnard, D. Bresser and C. Iojoiu, Energy Environ. Sci., 2018, 11, 3298-3309.

4 P. E. Stallworth, S. G. Greenbaum, F. Croce, S. Slane and M. Salomon, Electrochim. Acta, 1995, 40, 2137-2141.

5 F. Croce, S. D. Brown, S. G. Greenbaum, S. M. Slane and M. Salomon, Chem. Mater., 1993, 5, 1268-1272.

6 C. S. Kim and S. M. Oh, Electrochim. Acta, 2000, 45, 21012109.

7 J. Mindemark, M. J. Lacey, T. Bowden and D. Brandell, Prog. Polym. Sci., 2018, 81, 114-143.

8 O. Buriez, Y. B. Han, J. Hou, J. B. Kerr, J. Qiao, S. E. Sloop, M. Tian and S. Wang, J. Power Sources, 2000, 89, 149-155.
9 K. Kimura, J. Motomatsu and Y. Tominaga, J. Phys. Chem. C, 2016, 120, 12385-12391.

10 I. Y. EvchukR, I. Musii, R. G. Makitra and R. E. Pristanskii, Russ. J. Appl. Chem., 2005, 78, 1576-1580.

11 D.-W. Kim, B.-K. Oh and Y.-M. Choi, Solid State Ionics, 1999, 123, 243-249.

12 A. Hosseinioun and E. Paillard, J. Membr. Sci., 2019, under revision.

13 L. Edman, J. Phys. Chem. B, 2000, 104, 7254-7258.

14 D. M. Seo, O. Borodin, S.-D. Han, P. D. Boyle and W. A. Henderson, J. Electrochem. Soc., 2012, 159, A1489A1500.

15 N. A. Stolwijk, J. Kösters, M. Wiencierz and M. Schönhoff, Electrochim. Acta, 2013, 102, 451-458.

16 M. Kunze, Y. Karatas, H. D. Wiemhöfer and M. Schönhoff, Macromolecules, 2012, 45, 8328-8335.

17 M. Gouverneur, J. Kopp, L. van Wüllen and M. Schönhoff, Phys. Chem. Chem. Phys., 2015, 17, 30680-30686.

18 F. Hallberg, I. Furó and P. Stilbs, J. Am. Chem. Soc., 2009, 131, 13900-13901.

19 M. Giesecke, G. Mériguet, F. Hallberg, Y. Fang, P. Stilbs and I. Furó, Phys. Chem. Chem. Phys., 2015, 17, 3402-3408.

20 M. Brinkkötter, G. A. Giffin, A. Moretti, S. Jeong, S. Passerini and M. Schönhoff, Chem. Commun., 2018, 54, 4278-4281.

21 M. Gouverneur, F. Schmidt and M. Schönhoff, Phys. Chem. Chem. Phys., 2018, 20, 7470-7478.

22 M. Rosenwinkel and M. Schönhoff, J. Electrochem. Soc., 2019, 166(10), A1977-A1983.

23 O. Borodin, G. D. Smith and W. Henderson, J. Phys. Chem. B, 2006, 110, 16879-16886.

24 V. Lesch, Z. Li, D. Bedrov, O. Borodin and A. Heuer, Phys. Chem. Chem. Phys., 2016, 18, 382-392.

25 K. Oldiges, D. Diddens, M. Ebrahiminia, J. B. Hooper, I. Cekic-Laskovic, A. Heuer, D. Bedrov, M. Winter and G. Brunklaus, Phys. Chem. Chem. Phys., 2018, 20, 1657916591.

26 N. Molinari, J. P. Mailoa and B. Kozinsky, Chem. Mater., 2018, 30, 6298-6306.

27 O. Borodin and G. D. Smith, Macromolecules, 2006, 39, 16201629.

28 D. Diddens, A. Heuer and O. Borodin, Macromolecules, 2010, 43, 2028-2036.

29 D. Diddens and A. Heuer, ACS Macro Lett., 2013, 2, 322-326. 30 D. Diddens, E. Paillard and A. Heuer, J. Electrochem. Soc., 2017, 164, E3225-E3231.

31 M. A. Webb, B. M. Savoie, Z.-G. Wang and T. F. Miller III, Macromolecules, 2015, 48, 7346-7358.

32 S. Mogurampelly, O. Borodin and V. Ganesan, Annu. Rev. Chem. Biomol. Eng., 2016, 7, 349-371.

33 V. Sethuraman, S. Mogurampelly and V. Ganesan, Soft Matter, 2017, 13, 7793-7803.

34 O. Borodin and G. D. Smith, J. Phys. Chem. B, 2006, 110, 6279-6292.

35 O. Borodin and G. D. Smith, J. Phys. Chem. B, 2006, 110, 6293-6299.

36 O. Borodin, J. Phys. Chem. B, 2009, 113, 11463-11478. 
37 S. Jeschke, H.-D. Wiemhöfer and C. Mück-Lichtenfeld, Phys. Chem. Chem. Phys., 2014, 16, 14236-14243.

38 O. Borodin, M. Olguin, P. Ganesh, P. R. C. Kent, J. L. Allen and W. A. Henderson, Phys. Chem. Chem. Phys., 2016, 18, 164-175.

39 N. Chapman, O. Borodin, T. Yoon, C. C. Nguyen and B. L. Lucht, J. Phys. Chem. C, 2017, 121, 2135-2148.

40 G. Bieker, D. Diddens, M. Kolek, O. Borodin, M. Winter, P. Bieker and K. Jalkanen, J. Phys. Chem. C, 2018, 122, 21770-21783.

41 F. Schmidt, A. Pugliese, C. S. A. Mele and M. Schönhoff, 2019, manuscript in preparation.

42 M. Frisch, G. Trucks, H. Schlegel, G. Scuseria, M. Robb, J. Cheeseman, G. Scalmani, V. Barone and G. Petersson, Gaussian 16, Wallingford CT, 2016.

43 L. A. Curtiss, P. C. Redfern and K. Raghavachari, J. Chem. Phys., 2007, 127, 124105.
44 S. Grimme, S. Ehrlich and L. Goerigk, J. Comput. Chem., 2011, 32, 1456-1465.

45 A. V. Marenich, C. J. Cramer and D. G. Truhlar, J. Phys. Chem. B, 2009, 113, 6378-6396.

46 R. Bhandary and M. Schönhoff, Electrochim. Acta, 2015, 174, 753-761.

47 D. Battisti, G. A. Nazri, B. Klassen and R. Aroca, J. Phys. Chem., 1993, 97, 5826-5830.

48 D. W. McOwen, D. M. Seo, O. Borodin, J. Vatamanu, P. D. Boyle and W. A. Henderson, Energy Environ. Sci., 2014, 7, 416-426.

49 K. Hayamizu, Y. Aihara, S. Arai and W. S. Price, Solid State Ionics, 1998, 107, 1-12.

50 A. Munar, A. Andrio, R. Iserte and V. Compañ, J. Non-Cryst. Solids, 2011, 357, 3064-3069.

51 C.-K. Lin and I. D. Wu, Polymer, 2011, 52, 4106-4113.

52 I. D. Wu and F.-C. Chang, Polymer, 2007, 48, 989-996. 